\title{
Takotsubo Cardiomyopathy and Subsequent Seizures Induced by Flexible Bronchoscopy
}

\author{
Shion Miyoshi MD, Yuichiro Takeda MD PhD, Shoki Ro MD, Haruna Masaki MD, \\ Masayuki Hojo MD, and Haruhito Sugiyama MD PhD
}

\begin{abstract}
Takotsubo cardiomyopathy is a transient left-ventricular dysfunction that typically occurs in elderly women due to emotional or physical stress. An 85-y-old woman underwent flexible bronchoscopy to evaluate her malignant lymphoma. After flexible bronchoscopy, she experienced takotsubo cardiomyopathy diagnosed by left ventriculography and subsequent generalized tonic-clonic seizure diagnosed by electroencephalography. Magnetic resonance imaging of her brain after $1 \mathrm{~d}$ showed post-convulsive encephalopathy. We believe that these 2 consecutive incidents were caused by the physical stress of the flexible bronchoscopy. Key words: takotsubo cardiomyopathy; seizures; encephalopathy; bronchoscopy; complication; lidocaine. [Respir Care 2015;60(9):e151-e154. (C) 2015 Daedalus Enterprises]
\end{abstract}

\section{Introduction}

Takotsubo cardiomyopathy is an acute cardiac syndrome characterized by the sudden onset of chest pain or dyspnea with ST-segment elevation and transient left-ventricular dysfunction. It is thought to be a type of stunned cardiomyopathy in which the specific shape of the left ventricle resembles an octopus pot in a cardiac echogram or left ventriculogram. This syndrome occurs following emotional or physical stress. Recently, several cases of takotsubo cardiomyopathy associated with epileptic seizures have been reported. ${ }^{1-3}$ However, adverse events associated with bronchoscopy are relatively rare, and severe complications such as cardiovascular events or seizures are rarer still., ${ }^{4,5}$ This report concerns a patient with takotsubo cardiomyopathy that was induced by flexible bronchoscopy and the subsequent seizures that occurred.

\footnotetext{
The authors are affiliated with the Department of Pulmonary Medicine, National Center for Global Health and Medicine, Tokyo, Japan.

The authors have disclosed no conflicts of interest.

Correspondence: Shion Miyoshi MD, Department of Pulmonary Medicine, National Center for Global Health and Medicine, 1-21-1 Toyama Shinjuku, Tokyo 162-8655, Japan. E-mail: shion728532@ yahoo.co.jp.
}

DOI: $10.4187 /$ respcare. 03650

\section{Case Report}

An 85-y-old female suffering from mucosa-associated lymphoid tissue lymphoma of the lung was being treated by observation only beginning July 2010 . Because the mass lesion of the left upper lobe was growing, she underwent flexible bronchoscopy in April 2012 to evaluate the histologic transformation of the lymphoma. Before bronchoscopy, her body weight was $51.6 \mathrm{~kg}$, her blood pressure was $117 / 61 \mathrm{~mm} \mathrm{Hg}$, her heart rate was 87 beats/min, and oxygen saturation on room air was $96 \%$. Her electrocardiogram (ECG) was normal. She was given a premedication consisting of $0.5 \mathrm{mg}$ of atropine administered intramuscularly, $25 \mathrm{mg}$ of hydroxyzine hydrochloride administered intramuscularly, and $2 \%$ lidocaine administered by gargling. The patient then received intravenous conscious sedation with $1.2 \mathrm{mg}$ of midazolam. After airway examination with a fiberoptic bronchoscope, she underwent a transbronchial lung biopsy and brushing with endobronchial ultrasonography and a guide sheath. Topical anesthesia was administrated by transtracheal spray and splashing of $2 \%$ lidocaine, and the total lidocaine dose was $500 \mathrm{mg}$. Her oxygen saturation was maintained at a safe level during the procedures.

However, after $40 \mathrm{~min}$, near the end of the procedure, she became unconscious and pulseless in the peripheral artery, and her heart rate dropped to 30 beats $/ \mathrm{min}$. Bronchoscopic examination was discontinued as quickly as possible. Bradycardia immediately improved within a few sec- 

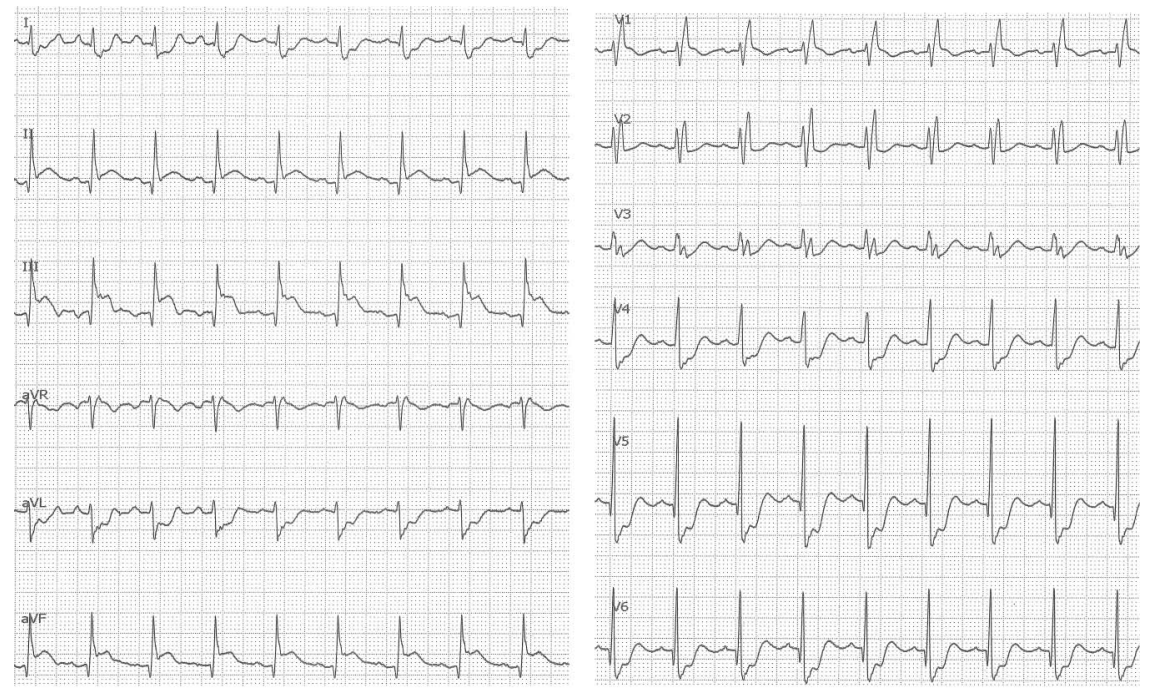

Fig. 1. Electrocardiogram showing ST-segment elevation in leads II, III, and augmented vector foot at the end of bronchoscopy.
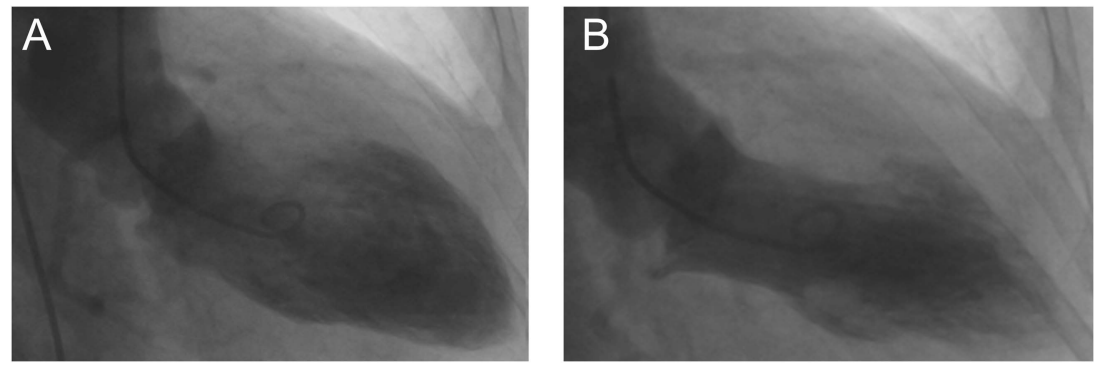

Fig. 2. Left ventriculogram showing mid-apical hypokinesis and basal hyperkinesis known as takotsubo cardiomyopathy. A: End-diastolic frame. B: End-systolic frame.

onds after intravenous atropine administration. There were no electrolyte imbalances and no metabolic function abnormality. Because we suspected acute myocardial infarction or stunned cardiomyopathy, we obtained an ECG, which showed ST-segment elevation in leads II, III, and augmented vector foot, and serum troponin I was elevated to $4.74 \mathrm{ng} / \mathrm{mL}$ immediately following the bronchoscopic procedure (Fig. 1). Although there was no evidence of obstructive coronary artery disease or coronary vasospasm as determined by coronary angiography, the left ventriculogram showed mid-apical hypokinesis and basal hyperkinesis, which are typical indicators of takotsubo cardiomyopathy (Fig. 2).

The patient was admitted to the ICU. Both brain magnetic resonance imaging (MRI) and magnetic cerebral angiography showed only old chronic ischemic changes (Fig. $3 \mathrm{~A}$ ), although she remained unconscious. She briefly regained consciousness after $4 \mathrm{~h}$ but then fell unconscious again after $8 \mathrm{~h}$ and also began to exhibit tonic-clonic seizure activity. She was given anticonvulsants, including diazepam, phenytoin, and propofol, and returned to consciousness without seizures with this drug treatment after
$12 \mathrm{~h}$. The electroencephalogram showed a kind of generalized seizure disorder (Fig. 4). On day 2 after admission, an MRI of her brain showed T2-prolonged lesions in the right frontal and parietal-occipital lobes (Fig. 3B), indicative of post-convulsive encephalopathy.

The patient's left-ventricular systolic function in the echocardiogram returned to normal on day 3. Up to day 22 , electroencephalograms continued to show marked improvement. On day 33, she was transferred to a rehabilitation hospital. An MRI of her brain after 5 months showed that the T2-prolonged lesions had improved markedly (Fig. 3C). Although she was still using anticonvulsants, she had no further epileptic seizures after discharge from our hospital.

\section{Discussion}

Takotsubo cardiomyopathy is characterized by transient left-ventricular dysfunction, ECG changes, and minimal elevation of cardiac markers mimicking the presentation of acute coronary syndrome. It is considered to be a kind of stunned cardiomyopathy. It occurs mainly in elderly 

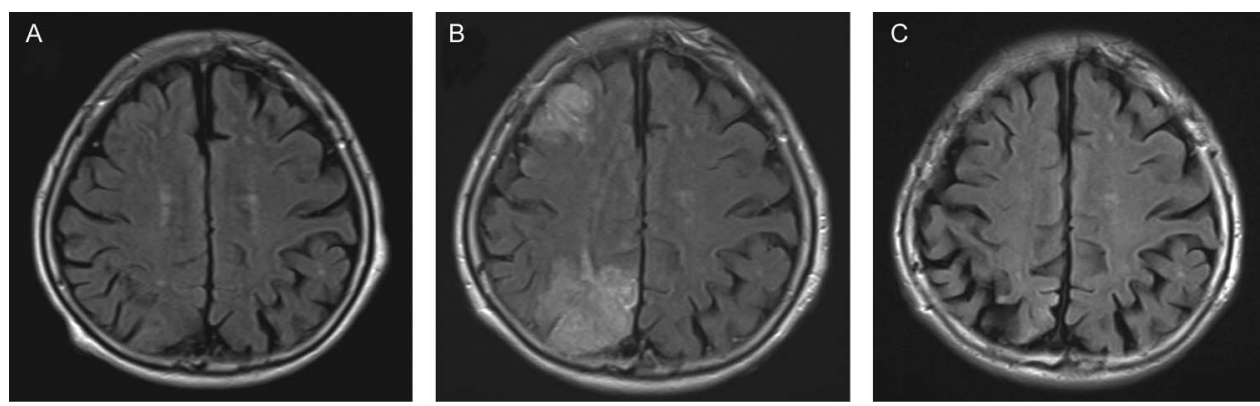

Fig. 3. A: T2-weighted-fluid-attenuated inversion recovery (T2-FLAIR) magnetic resonance imaging (MRI) showing only chronic ischemic changes. B: On day 2 of admission, MRI showed T2-FLAIR-prolonged lesions in the right frontal and parietal-occipital lobes. C: A 5-month follow-up brain MRI showed marked improvement of T2-FLAIR-prolonged lesions.

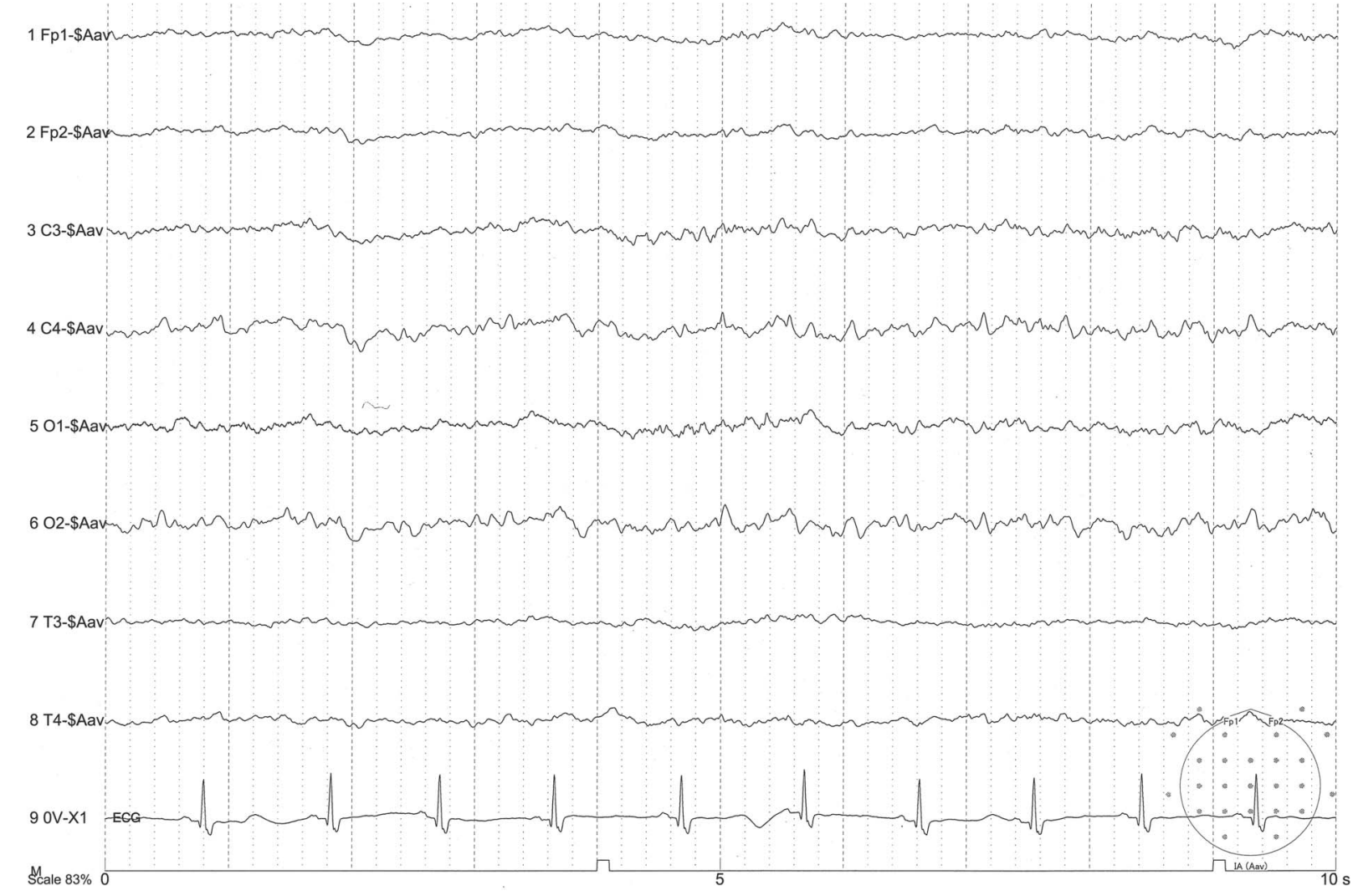

Fig. 4. Electroencephalogram demonstrating sharp waves maximal over the right occipital region.

women who have a history of physical or psychological stress. ${ }^{6,7}$ Coronary angiography is usually necessary to confirm that there is no evidence of significant coronary artery disease. 6,7 The pathophysiology of Takotsubo cardiomyopathy is unknown. Abnormal catecholamine releases are likely to play a central role.

Various cardiac complications such as arrhythmias have been reported during bronchoscopy ${ }^{4,5,8}$; however, takotsubo cardiomyopathy or seizures are uncommon. Only a few similar cases have been reported.9-12 In this case, takotsubo cardiomyopathy occurred due to physical stress from a bronchoscopic examination. A temporary lack of oxygen in her brain due to cardiogenic shock caused by the takotsubo cardiomyopathy is thought to have caused the seizures.

Seizure is a common complication in patients with metastatic brain tumors. ${ }^{13}$ However, in this case, there was no significant relationship between the seizures and the patient's malignant lymphoma because an MRI of her brain showed no metastatic lesions.

It has been reported that cardiac and neurologic toxicity can occur if the total dose of lidocaine exceeds $7 \mathrm{mg} / \mathrm{kg}$. ${ }^{14}$ Earlier studies showed that most patients have a peak plasma concentration well below the toxic level even with high 


\section{FleXible Bronchoscopy-Induced TAKotsubo CARdiomyopathy}

doses of topical lidocaine, ${ }^{15,16}$ so the relationship between seizure and topical lidocaine is considered to be obscure. Because the total dose of topical anesthesia was $500 \mathrm{mg}$ $(9.7 \mathrm{mg} / \mathrm{kg})$, it is probable that the high dose induced her cardiac event or seizures.

Midazolam is one of the benzodiazepines that have anticonvulsant action. Her seizures were delayed because midazolam kept them under control during bronchoscopy.

This is the first report of takotsubo cardiomyopathy and subsequent seizures induced by flexible bronchoscopy. Such severe complications should not be underestimated. Because the incidence of such complications is uncommon and unpredictable, it is especially important when the patient is elderly to prepare appropriate management such as routine ECG and saturation monitoring during examination.

\section{REFERENCES}

1. Cunnington C, Garg S, Balachandran KP. Seizure-associated takotsubo cardiomyopathy presenting with unheralded ventricular fibrillation. Int J Cardiol 2012;162(1):e21-e23

2. Bosca ME, Valero C, Pareja AI, Bonet M, Bosca I, Sanchez-Roy R, Ruvira J. Eur J Neurol 2008;15(5):e34-e35

3. Stöllberger C, Wegner C, Finsterer J. Seizure-associated Takotsubo cardiomyopathy. Epilepsia 2011;52(11):e160-e167

4. Credle WF Jr, Smiddy JF, Elliott RC. Complications of fiberoptic bronchoscopy. Am Rev Resp Dis 1974;109(1):67-72.

5. Suratt PM, Smiddy JF, Gruber B. Deaths and complications associated fiberoptic bronchoscopy. Chest 1976;69(6):747-751.

6. Gianni M, Dentali F, Grandi AM, Sumner G, Hiralal R, Lonn E. Apical ballooning syndrome or takotsubo cardiomyopathy: a systematic review. Eur Heart J 2006;27(13):1523-1529.
7. Sharkey SW, Lesser JR, Zenovich AG, Maron MS, Lindberg J, Longe TF, Maron BJ. Acute and reversible cardiomyopathy provoked by stress in women from the United States. Circulation 2005; 111(4):472-479.

8. Davies L, Mister R, Spence DP, Calverley PM, Earis JE, Pearson MG. Cardiovascular consequences of fiberoptic bronchoscopy. Eur Respir J 1997;10(3):695-698.

9. Guerrero J, Majid A, Ernst A. Cardiogenic shock secondary to Takotsubo syndrome after debridement of malignant endobronchial obstruction. Chest 2009;135(1):217-220.

10. Ok KS, Song BG, Park KS, Jung HG, Jung HJ, Park IN, et al. Inverted Tako-Tsubo cardiomyopathy associated with bronchoalveolar lavage. Heart Lung Circ 2011;20(7):476-478.

11. Wu FL, Razzaghi A, Souney PF. Seizure after lidocaine for bronchoscopy: case report and review of the use of lidocaine in airway anesthesia. Pharmacotherapy 1993;13(1):72-78.

12. Kumar S, Nath A, Singh S, Bhatia T, Kapoor A. An unusual complication during bronchoscopy: hypotension, global ST segment elevation and acute severe left ventricular systolic dysfunction. Respir Care 2013;58(9):e111-e115.

13. van Breemen MS, Wilms EB, Vecht CJ. Epilepsy in patients with brain tumours: epidemiology, mechanisms, and management. Lancet Neurol 2007;6(5):421-430.

14. Wahidi MM, Jain P, Jantz M, Lee P, Mackensen GB, Barbour SY, et al. American College of Chest Physicians consensus statement on the use of topical anesthesia, analgesia, and sedation during flexible bronchoscopy in adult patients. Chest 2011;140(5):13421350 .

15. Efthimiou J, Higenbottam T, Holt D, Cochrane GM. Plasma concentrations of lignocaine during fibreoptic bronchoscopy. Thorax 1982;37(1):68-71.

16. Loukides S, Katsoulis K, Tsarpalis K, Panagou P, Kalogeropoulos N. Serum concentrations of lignocaine before, during and after fiberoptic bronchoscopy. Respiration 2000;67(1):13-17. 